\title{
Mediating between AI and Highly Specialized Users
}

\author{
Daniela Petrelli, Aba-Sah Dadzie, \\ and Vitaveska Lanfranchi
}

\begin{abstract}
We report part of the design experience gained in X-Media, a system for knowledge management and sharing. Consolidated techniques of interaction design (scenario-based design) had to be revisited to capture the richness and complexity of intelligent interactive systems. We show that the design of intelligent systems requires methodologies (faceted scenarios) that support the investigation of intelligent features and usability factors simultaneously. Interaction designers become mediators between intelligent technology and users and have to facilitate reciprocal understanding.
\end{abstract}

A innovation, cases of successfully combining AI and humancomputer interaction (HCI) do exist, for example, Amazon's recommender. However, which design process should be followed to achieve such success is not clear: is a user-centered system design process enough, or should a new practice be developed to address the specificity of systems able to take autonomous decisions? ${ }^{1}$

In the context of the X-Media ${ }^{2}$ project, a large multisite EU project aiming at studying technology for knowledge management, sharing, and reuse, we started applying user-centered design but discovered standard practice needed to be modified to support effective communication between users and AI experts. From the very beginning it was clear that a participatory approach with both users and technologists discussing and contributing to the system design was not an easy goal: ambiguity in terminology and gaps in understanding could not be easily overcome.

A new role had to be devised, that of a mediator that moves between the two parties, facilitates the communication, and helps each group see the potential in what the other has to offer. A number of tools to facilitate the mediation and preserve the original intended meaning (so as to avoid "translation mistakes" while moving from one group to the other) were devised.

Mediating between the parties meant an increase in the number of design iterations, as, for example, discussing with users a potential solution generated new ideas for additional intelligent features that had to be discussed with the AI experts and then validated with users.

The rest of this article presents and discusses this experience in more detail. Reflecting on it, the time and effort needed to design an intelligent interactive system (IIS) adopting a user- 
centered design approach was higher than what a traditional interactive system would require. It was difficult to determine when the design should be stopped and the implementation started as new ideas and more advanced AI features continued to emerge at every meeting with users and AI experts.

\section{Users and Developers Focus Group Failure}

The goal of X-Media is to study, design, implement, and evaluate technology for the sharing and reuse of knowledge originated from disparate sources: text (generally technical reports), images (pictures taken in the workshops), and sensor data (from continuous monitoring of phenomena). The context of use is the manufacturing industry, in particular mechanical engineering (Fiat, the Italian car company) and aerospace engineering (RollsRoyce plc, UK manufacturer of gas turbines).

After a small number of use cases were selected by the users' representatives in the X-Media consortium, a number of workshops were set up in Rolls-Royce and Fiat to promote mutual understanding between the different expertise of the 16 X-Media partners. We aimed at collecting users' requirements with the help of the final users and jointly define which features the system should provide. We very quickly found out that to have AI specialists and aerospace or mechanical engineers in the same room is not enough to guarantee communication and mutual understanding.

The first problem was the terminology used by both parties. Users and AI experts used the same term to mean completely different concepts. The level of confidence is one such example: while users meant the confidence an aerospace engineer has in formulating a hypothesis on the potential cause of an observed phenomenon, AI used it to mean the confidence the system has in reasoning or a decision taken. This mismatch was not identified at first as both parties did not need an explanation for a term they considered well known.

A second, more radical problem, lay in the difficulties in understanding each others' point of view that prevented effective collaboration. AI experts in knowledge extraction and representation were not able to see how their technology could help users with their problem-solving tasks; users were unable to envisage an intelligent system that extracts knowledge from different sources, collates this, and proposes solutions. Their positions were just too far apart and could not be easily reconciled.

A mediator was needed, a designer of interactive intelligent systems (IISD) able to envisage how and where intelligent technology could improve users' jobs and present it in terms simple enough for the users to understand. ${ }^{3}$ Simultaneously the creativity of AI technologists had to be stimulated and channeled toward useful applications. It is essential that intelligent techniques provide a true advantage to the user if they are to be perceived as worth having (Horvitz 2002). It is the role of the IISD to combine traditional and intelligent features to support the user's tasks with whichever interaction is most appropriate in the perspective of a usable system.

\section{Usability for AI}

In X-Media we wanted to assure control and transparency (Höök 2000); that is: (1) the user should be allowed to check and correct any mistake the system might have made (control), and (2) the system should show its internal mechanisms to the degree needed to understand why the system is behaving as it is (transparency). X-Media then cannot be a closed system that presents users with already packaged knowledge but should visualize in an easy-to-understand way why a certain assertion is considered true and more likely than another; allow users to track where a piece of evidence comes from (that is, link to all the documents the pieces of information were extracted from); allow users to override whatever decisions the system has taken; and allow users to contribute their personal knowledge. ${ }^{4}$

These guidelines do not affect the user interface alone. Imposing the traceability of the source of a piece of knowledge required the knowledge base to keep the connection with the original document and any modification that occurred thereafter; the user inspection requires the knowledge base to store data in a human-understandable format. Stating these essential guidelines oriented the design of the whole system toward a solution that was in essence different from the view, common among AI researchers, that the modeling of the user will build machines that are cognitively compatible with humans (Winograd 2006) with no real need for designing for usability. Instead in X-Media we focused on the interaction between the human and the system and explicitly designed AI features for usability.

\section{Faceted Scenario and Vision Demonstrator}

A scenario-based design was adopted. A scenario is a story describing a person with specific characteristics and motivations who performs a specific task by interacting with a specific system (Carroll 1997). Because it is essentially a narrative, scenarios are easily understood and can effectively support discussions among the members of a design team (Carroll 1997). We have revisited the basic idea of scenarios, a narrative of a specific user interacting with a system, to include all the facets of a 


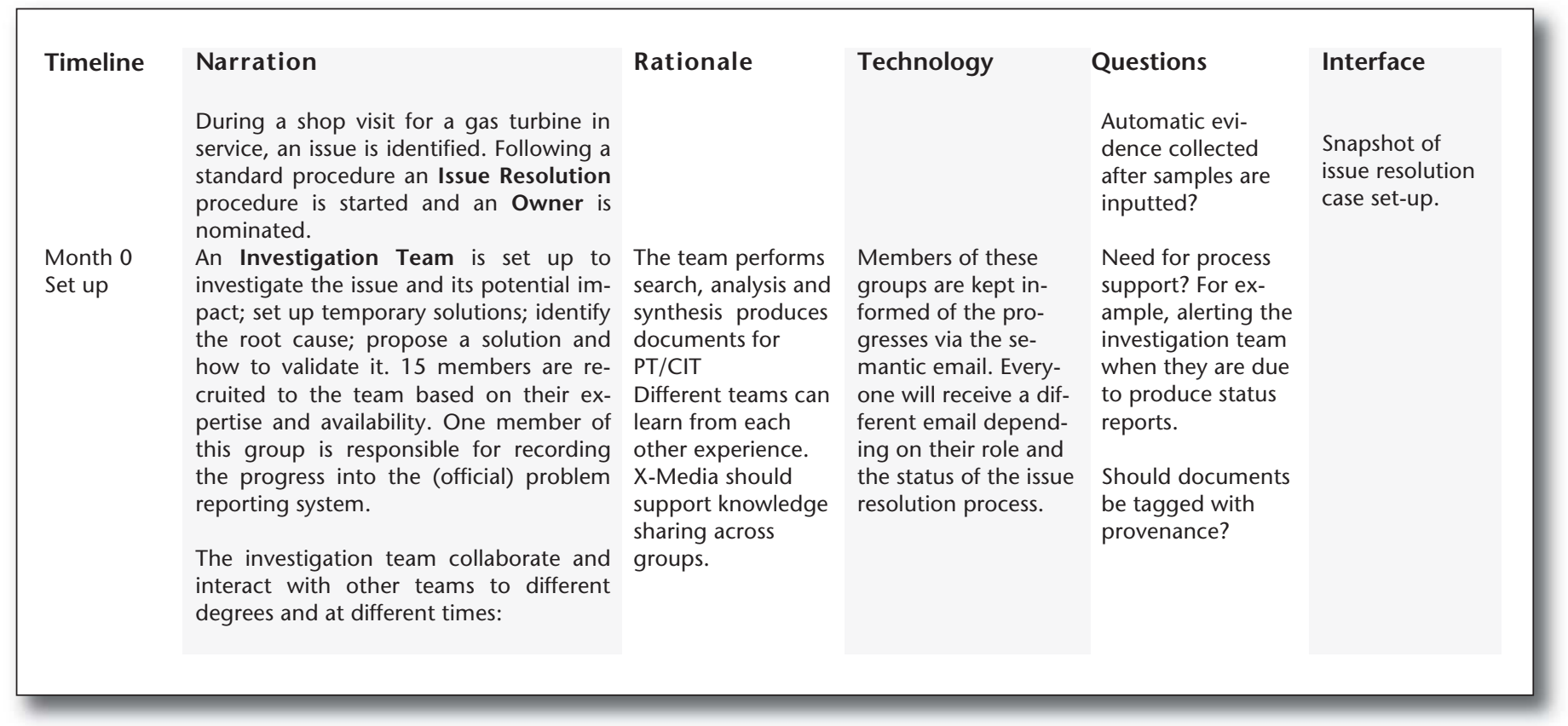

Figure 1. The Initial Passage of a Faceted Scenario.

complex discussion among partners (figure 1). The multiple aspects embedded in a faceted scenario allow different project members to look at the same story from different angles, that is, in a different column. Users concentrate on the story and can check whether the workflow described and tasks are realistic or not: being a narration, it allows users to contribute their own view. Interaction designers instead mainly focus on the rationale to make explicit their design choices. The technology column is where the contribution of $\mathrm{AI}$ is discussed and spelled out (figure 1). The initial scenario was written after a workshop in which a group of RollsRoyce engineers described their activities. This first scenario, only textual, reflected the designers' understanding of the user's task as contextualized with respect to the interaction with X-Media. In order to be an effective instrument of discussion, sketches were added to make the designers' vision more concrete.

Low-fidelity prototyping uses paper mock-ups or other forms of simulation that convey the sense of the final interaction (Catani and Biers 1998; Sefelin, Tscheligi, and Giller 2003; Virzi, Karis, and Sokolov 1996). We used paper mock-ups to illustrate scenarios and create a sort of storyboard. We refined this idea and paired the scenarios with a vision demonstrator, an interactive simulator of a working system ${ }^{5}$. It provided a simple but effective tool for communicating the vision of an improved process to end users and the project team alike. Figure 2 shows an example of part of the vision demonstrator that allowed the exploration of the concept of the knowledge cloud, a visual representation of the aggregated data that composes an assertion (background, figure 2). What this vision demonstrator simulates is the automatic extraction of information from different data sources (images, data tables, text) and their aggregation to a single concept.

A vision demonstrator shows how the system would work from the user's point of view. At the same time, it illustrates what the intelligent components are expected to produce and how the result will be presented to the user. In figure 2 , the ontology (on the left) is used to search the knowledge base by clicking concepts; this retrieves assertions (large panel on the right) displayed as an aggregation of heterogeneous clips such as snippets of text, images, tables, and graphs. The different sizes of the clips represent the relevance the system has given to that bit of evidence. By clicking a snippet, the single assertion is retrieved and visualized with its associated metadata, for example, provenance (foreground, figure 2). The user can then inspect the piece of knowledge, can add annotations or see those left by others, and can access the document that originally contained this image (example in top left). By inspecting the original document the user can better understand why the system has extracted this knowledge and can approve or reject the suggestion by clicking the + or - at the borders of the confidence-level bar. Approving or rejecting affects the contribution the assertion has to the probability of the hypothesis being analyzed. 


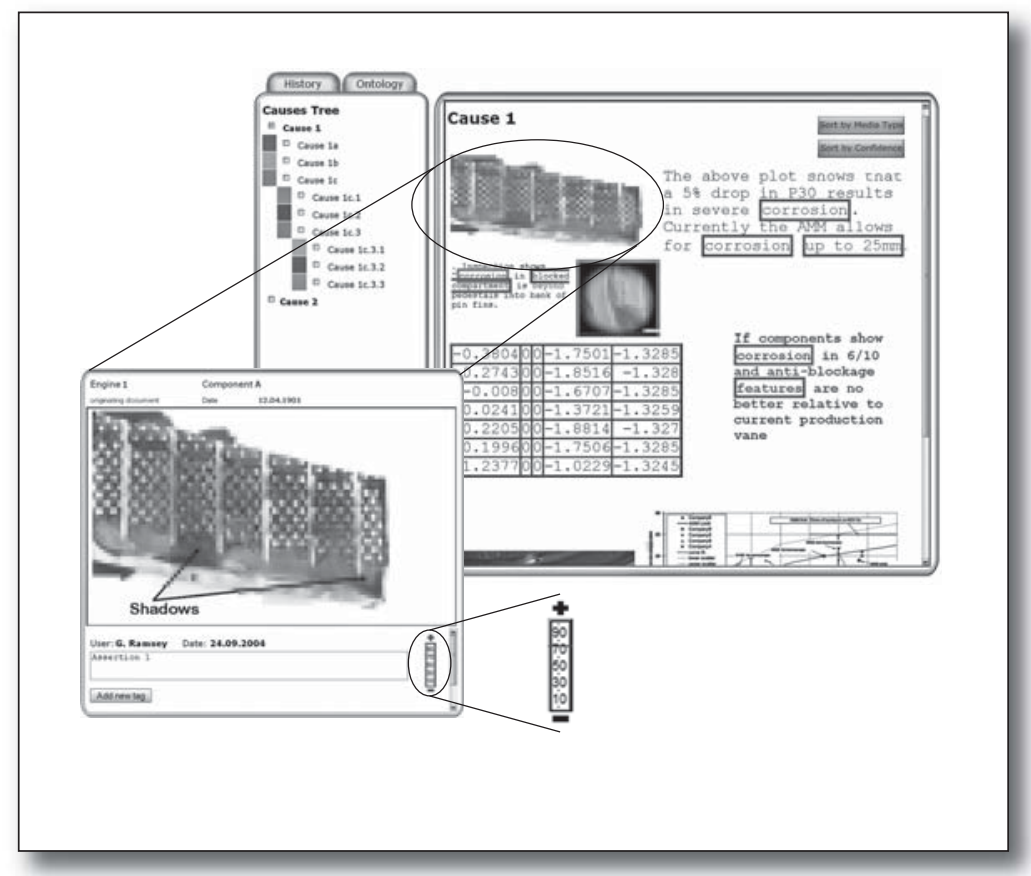

Figure 2: An Aggregation of Assertions as Seen by the User.

Knowledge cloud (background), the detailed information of an assertion (foreground left), and the confidence control.
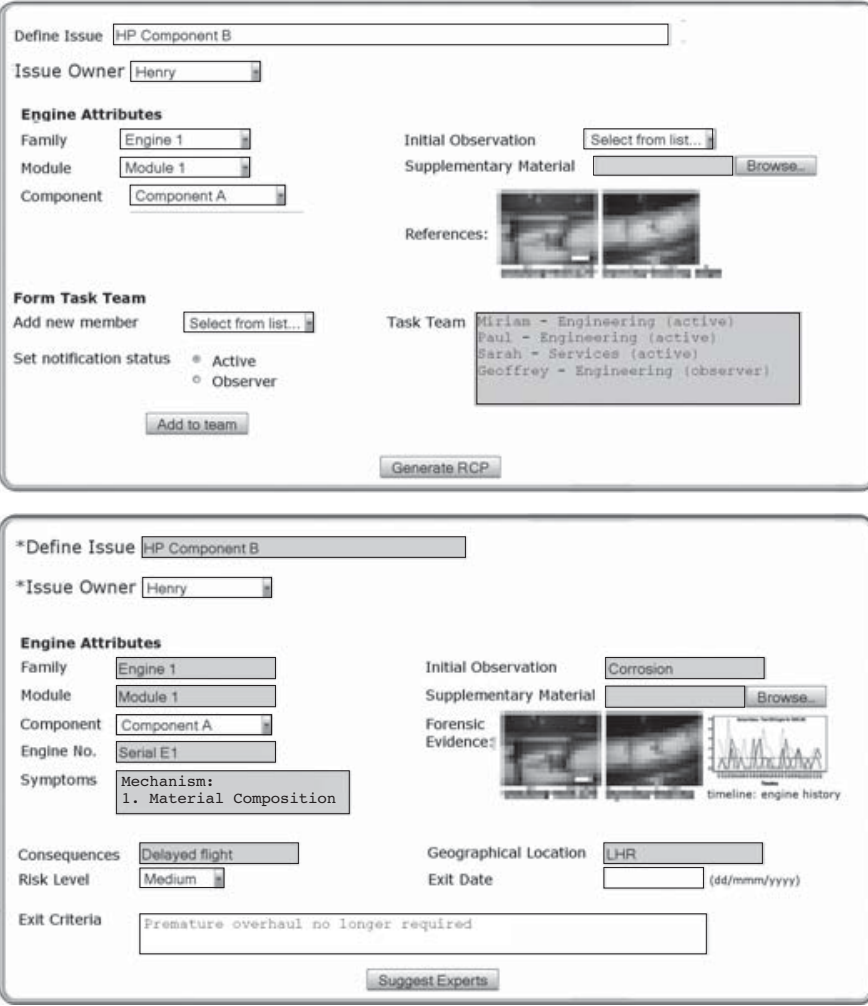

Figure 3. An Example of the Evolution of the Vision Demonstrator after the Validation of the Scenario with Users.
The vision demonstrator provides AI experts with a "golden standard" to aim at or at least a concrete view of the user's expectations. Independent AI modules are all combined and used in the user interface: the outcome of the information extraction process, the result of the knowledge fusion, the tracking of the provenance, and the feedback to the reasoning module.

From the final user's point of view the vision demonstrator shows what AI technology could do for them and allows them to imagine how this innovative technology could fit in with their working practices.

Following Hounde and Hill (1997), "prototypes provide the means for examining design problems and evaluating solutions." The vision demonstrator essentially prototypes the role and the look and feel of the final system, but the implementation is not considered. This proved to be a good tool for discussion among people with heterogeneous expertise, but the design team needed to constantly refer to it to ensure its functionalities would actually be implemented. Indeed there is the risk the solution selected in this phase would be forgotten once the implementation starts and the focus shifts from effective design to technical issues. The envisaged features might not be implementable as expected thus requiring a revision of the vision demonstrator that, in this way, acts as a common reference model.

\section{Mediating between User and AI Technologist}

The vision demonstrator was used for the validation of the design rationale in a participatory design session: users met interaction designers to discuss features and interaction of X-Media focusing on cases like the one described in the scenario. By discussing with users on the basis of concrete examples (scenarios) it was possible to generate new interaction ideas and explore new AI features, as can be seen by comparing the two mock-ups in figure 3.

New versions of the scenario and vision demonstrator were created to be discussed, this time, with the technical partners as the new layout could accommodate more intelligent functionalities. The outcome of this step was the introduction of technical insertion points (TIPs). In fact, AI technology often works in the background and might never surface at the user interaction level. TIPs support considering those technical aspects in the context of the whole user-system interaction as they provide anchor points for a deeper and more detailed description of the technical aspects behind the interface. In other words, as signposts, the TIPs highlighted the contribution of intelligent modules to the interaction (figure 4). This final schema 


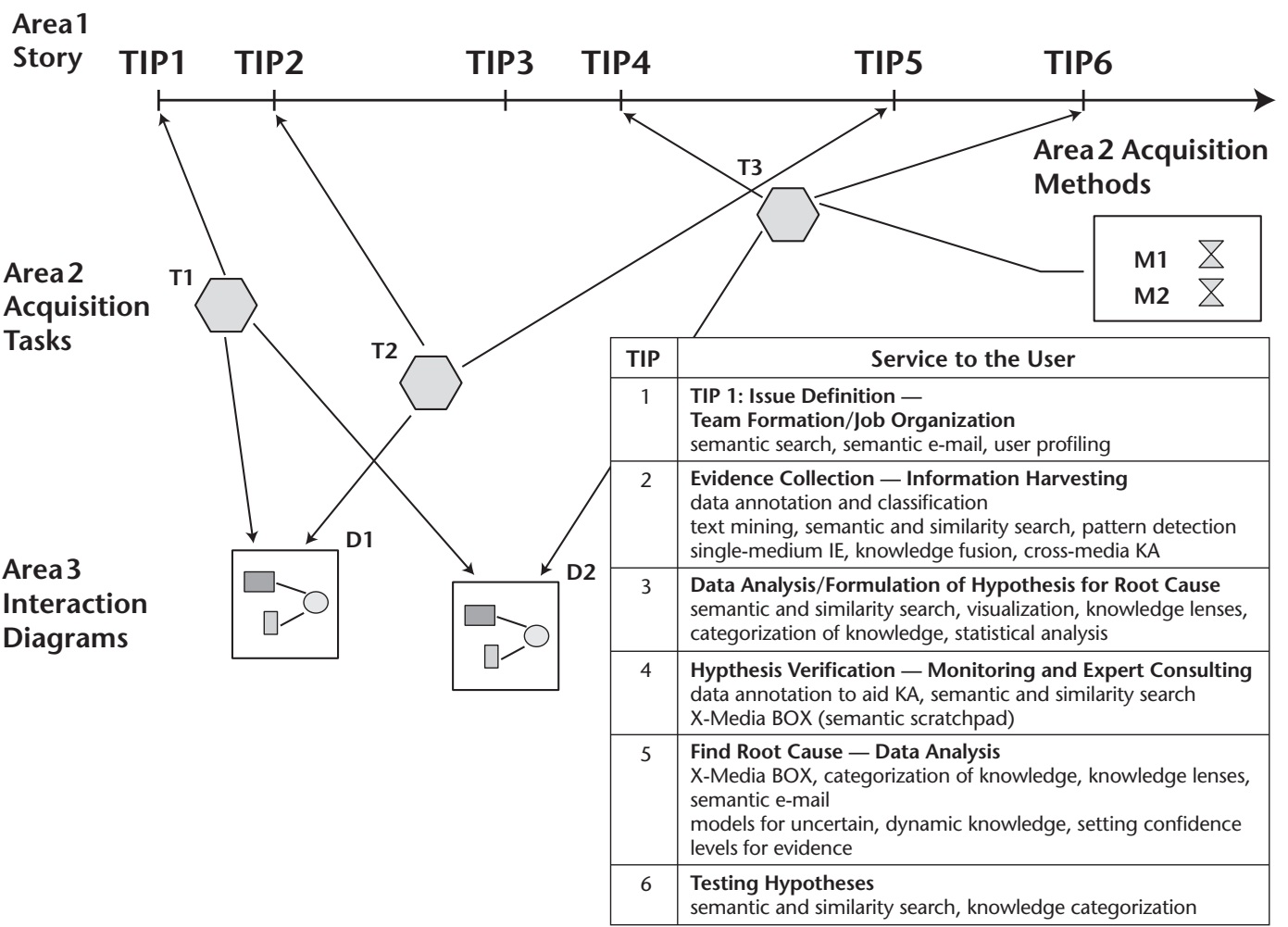

Figure 4. Contribution of Intelligent Modules to the Interaction.

An example of a map of the contribution of intelligent modules (the hexagons) to the overall interaction (top line) and the planned prototypes.

was both a representation of the user interaction and a plan for technology development. As such it allowed the different parties to autonomously work on their parts with a certain assurance of how those modules would be incorporated into the whole system. The next step for the IISD was to validate the current design with users.

\section{Design Validation}

Low-fidelity prototyping allows designers to explore alternative ideas at low cost. In X-Media the vision demonstrator was used to explore alternative solutions for how the knowledge should be presented to the user. To collect feedback on which visualizations were worth pursuing and which ones could be abandoned a collaborative evaluation session with engineers and designers was set up. The whole scenario was inspected and discussed.

Three possible views were considered at this point: a table-based organization, a decision tree (figure 5), and a knowledge graph (figure 6).

In previous meetings with users we were shown tables and trees as working tools; we therefore expected them to prefer simpler displays such as those in figure 5. However some advanced data visualizations were shown (by the designers): a semantic network to explore the relationships between assertions retrieved from the knowledge base (figure 6); a semantic filter to focus on subsets of data; and a parallel coordinates visualization that provides a temporal or geographical map through data.

To our surprise the more complex visualizations were the most preferred as it was possible for the user to explore and absorb more information at a single glance. Engineers are accustomed to different plots and graphs and therefore did not have problems grasping the meaning of the visualization and the potential manipulation. The graphical solution was therefore selected for implementation (figure 6). 


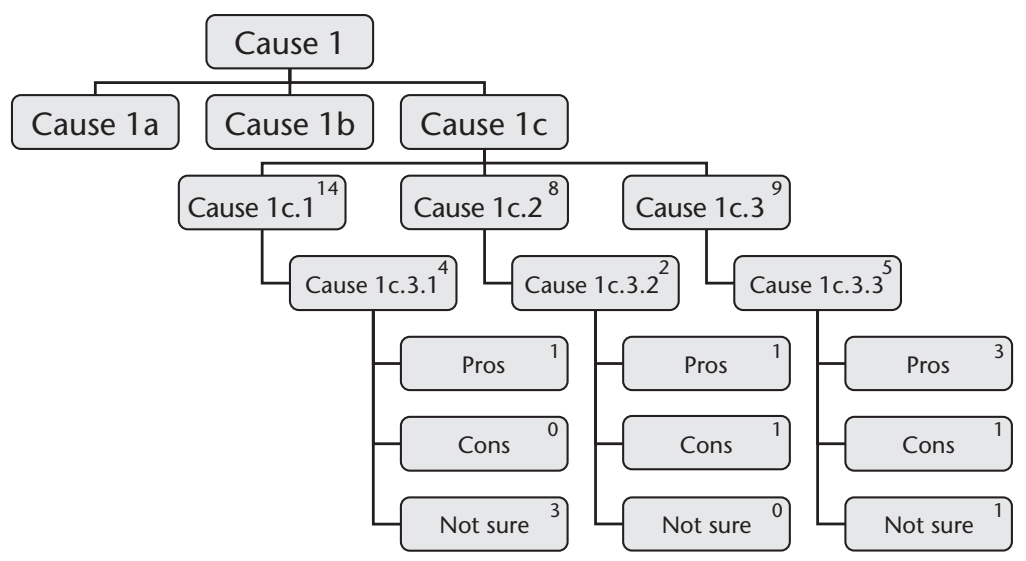

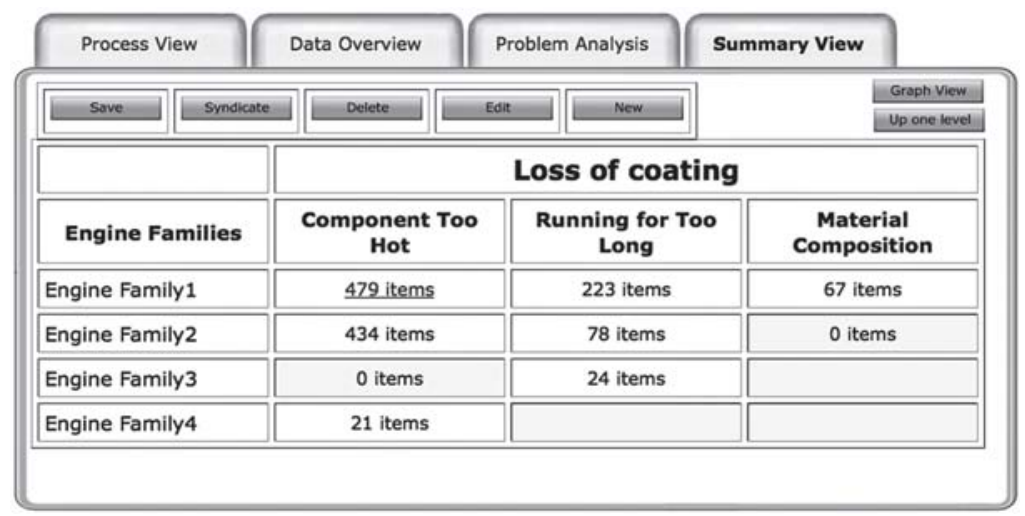

Figure 5. Alternative Knowledge Views.

Views using (top) a tree, and (bottom) a table.

Multiple graphical visualizations were, in the end, implemented. Indeed during the discussion with users it emerged that the complex problemsolving activity engineers are involved with requires different tools at different times for different purposes. The table provides an easy-to-understand summary of the current status of the analysis; the tree provides a general guidance on the investigation process (which branches have been considered and which still need attention); the graph shows connections among data not otherwise visible.

The final design includes several tools refined from this initial exploration.

An investigation tracking table is provided to organize the evidence collected to better contrast and compare facts. It also provides a summary of the investigation useful for newcomers to understand the current status. While users can benefit from using it as a way of rationalizing their thoughts, the X-Media system could use the information inserted by users in the table to reinforce its inference mechanisms as the assertions in the table can be considered true (that is with a confidence of 100 percent).

A root-cause analysis tree is used to represent the whole investigation space, as a visual way to collate evidence and counterevidence and direct the (human) reasoning towards the more likely causes.

A number of alternative visualizations, of which the knowledge graph is one, support knowledge exploration and manipulation.

\section{Design for AI Usability}

Interaction designers of IIS have to work to ensure the usability of the final system as user interface issues could affect the inner levels. In the first 
phases of the system conception they should provide guidelines to the team at large to assure the foundation for the system usability are in place. In the case of X-Media, for example, to assure transparency and control the architecture had to be purposefully designed to allow users to inspect the knowledge base and change values of assertions, or to create knowledge from scratch on the basis of spontaneous intuitions they may have had.

In designing usable IIS the main challenge is the negotiation between the different positions. We noticed that researchers specialized in intelligent algorithms were not accustomed to talking to users and initially failed in seeing where their technology could be useful. On the other hand, users could not always understand and appreciate the advantages of intelligent technology. To make the communication successful, interaction designers had to act as mediators between the two opposite positions.

This often meant several design iterations as new ideas were generated at each meeting that needed to be validated with the opposite party. For example, during initial interviews users showed compare-and-contrast tables they use as a summary during the investigation process. A table summary was included in the vision demonstrator; discussions that followed with AI experts resulted in them suggesting the new functionality of populating the summary table automatically. This led to a new iteration with users where the possibility of automatically filling the table was discussed and new requirements were produced, for example, graphically distinguishing in the table elements included by humans versus those added by the system.

The use of faceted scenarios and a vision demonstrator have been instrumental in making the communication effective and helping everyone contribute ideas and expertise to the development of the X-Media design. This proved how the design of an IIS should be a collective learning process where technology is challenged by real-world problems and users open their minds to new possibilities. Iterative cycles of usability analysis, user requirements, and functionality analysis promoted cross fertilization between HCI and AI: both views evolved and changed each other during the iterative design process.

To facilitate the integration of AI technology into the user interaction, the scenarios were enriched with technical insertion points, introduced to provide a deeper description of the technical aspects behind the interface. As signposts, the TIPs highlighted the contribution of intelligent modules to the interaction and allowed more precisely defining what the input/output of those modules should be to exploit AI technology and assure usability.

Besides new tools for the user-centered design of

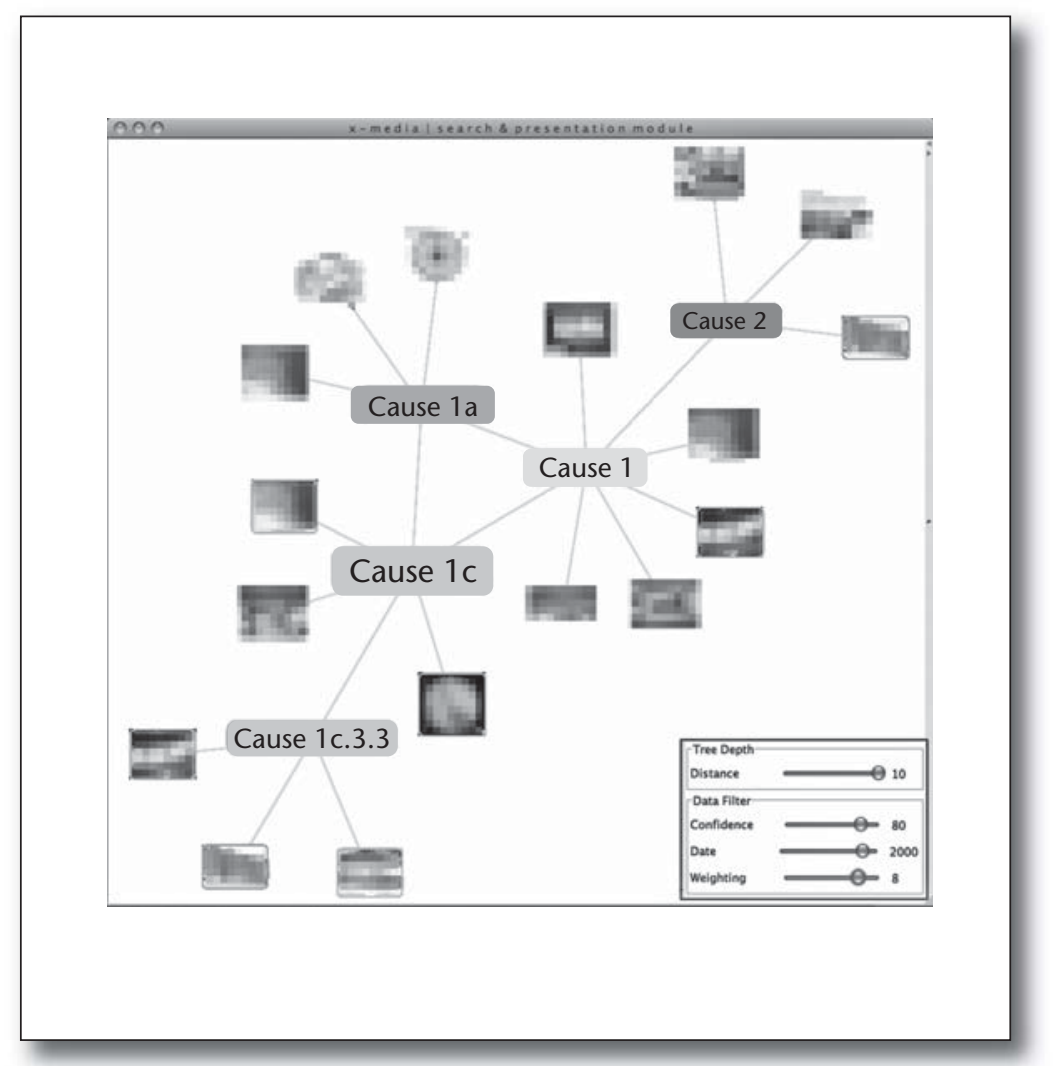

Figure 6. The Semantic Network Shows Relationships between Assertions in the Knowledge Base.

Node colors map to a concept in the ontology; size maps to the probability the assertion is correct. The graph is built using prefuse (Heer, Card, and Landay 2005) and query sliders (Ahlberg and Shneiderman 1992) to support dynamic searching to focus on subsets of data.

intelligent systems, a new figure has to be included in the team, that of an interaction designer aware of the possibilities AI offers as well as HCI methodologies and best practice. This figure has to mediate between the opposite positions of highly demanding users and AI experts. The design process has to be revisited to allow everyone to contribute their perspective in a mutually understandable way. To improve communication between users and AI experts both groups were considered as users by the interaction designers and the participatory design paradigm was used to work alternatively with both.

On reflection, the main lesson learned is that of starting the design with a "not so intelligent" version of the system and iteratively increasing complexity over different versions of the design. The reason for this claim is that gaining users' confidence in professional environments is very difficult. Those discretional users will accept new tools only if they see a clear benefit. The first clear ben- 
efit is a well-designed user interface/interaction with minimal intelligence that already improves on the tools they currently use. Intelligence can then be built on this platform as use increases and confidence grows. AI experts should be patient and wait to see where their contribution can better fit. The final outcome can be more rewarding as more innovative solutions could emerge through iterative redesign.

\section{Acknowledgement}

The research reported in this article is funded by the X-Media project, sponsored by the European Commission as part of the Information Society Technologies program under EC grant number ISTFP6-026978. We thank all the X-Media partners for the stimulating discussions. We are indebted to Andy Harrison, and Colin Cadas, Rolls-Royce plc, for their continuous support.

\section{Notes}

1. This is a major topic of the usability engineering theme article in this issue.

2. www.x-media-project.org.

3. As is discussed in the usability engineering theme article (Spaulding and Weber 2009), it is important for the designers of a system to understand user needs and the context in which they work. The mediator, as described here, is an excellent way to promote this understanding. 4. These issues are discussed in the usability side effects theme article with somewhat different terminology: in terms of the problems of inadequate comprehensibility and inadequate performance (including errors). Because various different terms have been used in the literature for many years in discussions of these issues, it may be impossible ever to achieve complete uniformity of terminology. For example, the authors here are using the terminology from an influential previously published article.

5. The vision demonstrator is a great example of a tool for conducting need validation and exploratory design, as discussed in the usability engineering theme article.

\section{References}

Ahlberg, C., and Shneiderman, B. 1992. AlphaSlider: A Compact and Rapid Selector. In Proceedings of the Tenth Conference on Human Factors in Computing Systems, 365371. New York: Association for Computing Machinery.

Carroll, J. M. 1997. Scenario-Based Design. In Handbook of Human-Computer Interaction, 2nd ed., ed. M. Helander, T. Landauer, and P. Prabhu, 384-406. Amsterdam: Elsevier Science B. V.

Catani, M. B., and Biers, D. W. 1998. Usability Evaluation and Prototype Fidelity: Users and Usability Professionals. In Proceedings of the Human Factors and Ergonomics Society Forty-Second Annual Meeting, 1331-1336. Santa Monica, CA: Human Factors and Ergonomics Society.

Heer, J.; Card, S.; and Landay, J. 2005. Prefuse: A Toolkit for Interactive Information Visualization. In Proceedings of the Twenty-Third Conference on Human Factors in Computing Systems, 421-430 . New York: Association for Computing Machinery.
Hounde, S., and Hill, C. 1997. What Do Prototypes Prototype? In Handbook of Human-Computer Interaction, 2nd ed., ed. M. Helander, T. Landauer, and P. Prabhu. Amsterdam: Elsevier Science B. V.

Höök, K. 2000. Steps to Take Before IUIs Become Real. Interacting with Computers 12(4) 409-426.

Horvitz, E. 2002. Principles of Mixed-Initiative User Interfaces. In Proceedings of the Twentieth Conference on Human Factors in Computing Systems, 1-8 . New York: Association for Computing Machinery.

Sefelin, R.; Tscheligi, M.; and Giller, V. 2003. Paper Prototyping-What Is It Good for? A Comparison of Paper and Computer-Based Prototyping. In Proceedings of the Twentieth Conference on Human Factors in Computing Systems, 778-779. New York: Association for Computing Machinery.

Virzi, R. A.; Karis, D.; and Sokolov, J. L. 1996. Usability Problem Identification Using Both Low- and High-Fidelity Prototypes. In Proceedings of the Fourteenth Conference on Human Factors in Computing Systems, 236-243. New York: Association for Computing Machinery.

Winograd, T. 2006. Shifting Viewpoints: Artificial Intelligence and Human-Computer Interaction. Artificial Intelligence 170(1): 1256-1258.

Daniela Petrelli is a lecturer (British equivalent of assistant professor) in the Department of Information Studies at the University of Sheffield, UK. She has published extensively in the area of human-computer interaction and information access, including multimedia, multilingual, personal digital memories, user-centered design and evaluation. Her area of expertise includes innovative methodologies for the design and evaluation of nontraditional systems, for example, intelligent interactive systems, mobile and adaptive devices, tangible personal technology. She coordinates the user-centered activity in the EU IP project X-Media on semantic-based knowledge management in manufacturing (VI Framework of the European Community).

Aba-Sah Dadzie is a research associate at the Department of Computer Science at the University of Sheffield. She has a Ph.D. in computer science, where she applied information visualization to data analysis in bioinformatics. She currently does research in human-computer interaction and visual analytics, with a focus on applications for knowledge management that take advantage of semantic web technologies. She coordinates a major industrial use case in the EU IP project X-Media and also worked on the UK DTI project IPAS.

Vitaveska Lanfranchi is a research fellow at the Department of Computer Science at the University of Sheffield. She has a Ph.D. in communication science, where she studied context-sensitive, intelligent mobile interaction. She currently does research in human-computer interaction for web intelligence and the semantic web, with a focus on knowledge management. She is currently the coordinator of the HCI and application activity of the OAK Lab. She is coinvestigator in the EU IP project WeKnowIt and has previously worked on the UK DTI project IPAS. 